\title{
An Improved Sobel Edge Detection Operator
}

\author{
Yao Lang ${ }^{1, a^{*}}$ and Dan Zheng ${ }^{1, b}$ \\ ${ }^{1}$ College of electronic and Information Engineering Shenyang Aerospace University, China \\ ashenhanglangyao@163.com, b24867289@qq.com
}

Keywords: Sobel edge detection; Gradient algorithm; Image edge; Anti-noise performance

\begin{abstract}
The paper proposes an improved gradient algorithm which is based on the existing sobel edge detection. The algorithm is not only simple, but also can solve the coordination problem between the detection precision and anti-noise performance. Experimental results prove that using the modified gradient algorithm to detect image edge is not only clear outline, good real-time, but also has better anti-noise performance.
\end{abstract}

\section{Introduction}

The edge is the basic characteristics of the image, and which contains a wealth of internal information [1]. The various detection methods in the existing do not have an absolute advantage; they also solve some problems at the same time corresponding with this or that deficiencies. For example, some algorithms have a strong anti-noise performance, but the detection accuracy is not high [2]. Contrary to improve detection accuracy and anti-noise performance is getting worse. Although some will be both to certain extent coordinated, but the algorithm becomes complicated [3], it makes the results of the implementation time lengthened.

In order to solve these contradictions, this paper puts forward an improved gradient algorithm, and verified the algorithm has the advantage in the anti-noise performance through simulation experiments.

\section{The Traditional Sobel Edge Detection Operator}

The traditional sobel operator comprises two groups of $3 \times 3$ matrixes [4,5], which only have the horizontal and vertical directions of the template, but there are many directions of the edge. In order to detect the edge of the eight directions such as 45,135 etc, it was suggested that the improved sobel edge detection operator [6], which adds six directions of the template based on the traditional sobel algorithm, so it becomes eight directions of the template as shown in Fig. 1. Fig. 2 is schematic diagram of the direction. Although the sobel edge detection operator takes eight directions of the template image convolution, it increases the edge detection of direction [6,7], but due to increase the computational complexity, the speed of edge detection is reduced.

$$
\left[\begin{array}{ccc}
-1 & -2 & -1 \\
0 & 0 & 0 \\
1 & 2 & 1
\end{array}\right]\left[\begin{array}{ccc}
-2 & -1 & 0 \\
-1 & 0 & 1 \\
0 & 1 & 2
\end{array}\right]\left[\begin{array}{lll}
-1 & 0 & 1 \\
-2 & 0 & 2 \\
-1 & 0 & 1
\end{array}\right]\left[\begin{array}{ccc}
0 & 1 & 2 \\
-1 & 0 & 1 \\
-2 & -1 & 0
\end{array}\right]
$$

Direction $1 \quad$ Direction $2 \quad$ Direction $3 \quad$ Direction 4

$$
\left[\begin{array}{ccc}
1 & 2 & 1 \\
0 & 0 & 0 \\
-1 & -2 & -1
\end{array}\right]\left[\begin{array}{ccc}
2 & 1 & 0 \\
1 & 0 & -1 \\
0 & -1 & -2
\end{array}\right]\left[\begin{array}{ccc}
1 & 0 & -1 \\
2 & 0 & -2 \\
1 & 0 & -1
\end{array}\right]\left[\begin{array}{ccc}
0 & -1 & -2 \\
1 & 0 & -1 \\
2 & 1 & 0
\end{array}\right]
$$

\section{Direction $5 \quad$ Direction $6 \quad$ Direction $7 \quad$ Direction 8}

Figure 1. Eight direction template 


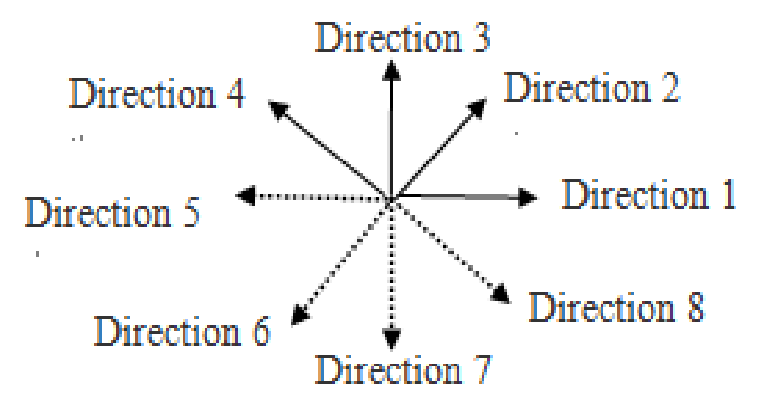

Figure 2. Schematic diagram of the direction

\section{Improved Sobel Edge Detection Operator}

The Paper Determines the Sobel Operator Direction of the Template. Based on the above eight direction template by careful research and analysis, this paper found in the vertical direction of the template on the pair wise, that is, direction 1 and direction 5, direction 2 and direction 6 , direction 3 and direction 7 , direction 4 and direction 8 , that take convolution of the gradient in the image of a pixel, the result opposite each other. So it can be reduced four template in the above eight templates, and then using the first four or after four templates take the absolute value of convolution results. This ensures that the output of results is the same as using eight templates to take convolution, but the computation can be reduced by nearly half.

This Paper Uses a Gradient Calculation Method. The traditional sobel algorithm is to take the maximum of convolution as the point of output value [8]. This paper presents a difference calculation method of gradient. It takes results of the absolute value difference between the maximum minus the minimum as the point of the gradient value. Because the point of edge has stronger gray mutation and directional, gradient value is larger along the edge direction, and the direction perpendicular to the edge is smaller, while the noise is random without direction, its gray mutation is weaker, the gradient value of which almost are the same in each direction. The difference calculation method of gradient for the edge point is similar to the traditional sobel algorithm [9], but in terms of noise has undergone great changes. The gradient difference equal of which will be equal to or approximately 0 , so that the algorithm improves the ability of resisting noise.

\section{Verify the Improved Operator}

Comparison of Anti-noise Performance. The following will use the gradient method proposed in this paper to test the two edges.Fig. 3 is the horizontal edge.Fig. 4 is the edge of curve or broken line.

\begin{tabular}{|c|c|c|}
\hline $\mathrm{X} 1$ & $\mathrm{X} 2$ & $\mathrm{X} 3$ \\
\hline $\mathrm{X} 4$ & $\mathrm{X} 0$ & $\mathrm{X} 5$ \\
\hline $\mathrm{X} 6$ & $\mathrm{X} 7$ & $\mathrm{X} 8$ \\
\hline
\end{tabular}

Figure 3. The horizontal edge

\begin{tabular}{|c|c|c|}
\hline $\mathrm{X} 1$ & $\mathrm{X} 2$ & $\mathrm{X} 3$ \\
\hline $\mathrm{X} 4$ & $\mathrm{X} 0$ & $\mathrm{X} 5$ \\
\hline $\mathrm{X} 6$ & $\mathrm{X} 7$ & $\mathrm{X} 8$ \\
\hline
\end{tabular}

Figure 4. The edge of curve or broken line

Assume that the gray value for the pixel point of the white block above diagram is ' 255 ', and the black block indicates that the gray value of pixel point is ' 0 '. After using the improved sobel template convolution operation.Eq. 1 is the maximum absolute value of gradient in the horizontal edge. Eq. 2 is the minimum absolute value of gradient. Eq. 3 is the gradient difference.

$$
\operatorname{Maxg}=|\mathrm{X} 1+\mathrm{X} 2+\mathrm{X} 3| \times 255=4 \times 255=1020
$$




$$
\begin{aligned}
& \text { Ming }=|\mathrm{X} 1+\mathrm{X} 2+\mathrm{X} 3| \times 255=0 \times 255=0 \\
& \text { Maxg }- \text { Ming }=1020-0=1020=\text { Maxg }
\end{aligned}
$$

Similarly for the vertical edges are equally Maxg-Ming = Maxg, that is, using the difference method to calculate the gradient is the same as the maximum gradient for straight edge.

For the Fig. 4, the edge of curve or broken line has the following results.Eq. 4 is the maximum absolute value.Eq. 5 is the minimum absolute value.Eq. 6 is the gradient difference.

$$
\begin{aligned}
& \text { Maxg }=|\mathrm{X} 3+\mathrm{X} 5| \times 255=3 \times 255=765 \\
& \text { Ming }=|\mathrm{X} 3+\mathrm{X} 5| \times 255=1 \times 255=255 \\
& \text { Maxg }- \text { Ming }=765-255=510 \approx \text { Maxg }
\end{aligned}
$$

That is, the method between using the difference to calculate the gradient and the maximum gradient value is similar for the edge of the curve or line.

The noise has no direction and its gradient value is almost no difference or little difference in each direction, the results using the difference calculation of the gradient should be Maxg-Ming $=0$ or $\approx 0$, which is different for using the maximum gradient value as output. Thus, the method proposed in this paper improves the ability of restraining noise than the original sobel algorithm through the verification of the theory.

Comparison of processing time. The following will be compared to the processing time between the improved sobel algorithm and sobel algorithm in the eight directions. Q-point is assumed to be a point in the image of processing [10]. Fig. 5 is the gray of $3 \times 3$ neighborhoods. When using the direction i-template of sobel operator to take process, the gray value of image for Q-point is set to $\mathrm{a}_{\mathrm{i}}(\mathrm{i}=1,2,3,4,5,6,7,8)$.After the eight templates processing, Eq. 7 is the gray value of Q-point.

$$
\left[\begin{array}{lll}
q_{0} & q_{1} & q_{2} \\
q_{3} & q_{q} & q_{4} \\
q_{5} & q_{6} & q_{7}
\end{array}\right]+
$$

Figure 5. The gray of $3 \times 3$ neighborhoods

$$
\mathrm{w}=\max \left\{\mathrm{a}_{\mathrm{i}}\right\}, \mathrm{i}=1,2,3,4,5,6,7,8
$$

For example, using the template of direction 1, it can get the following formula such as Eq. 8 .

$$
\mathrm{a}_{1}=-\mathrm{q}_{0}-2 \mathrm{q}_{1}-\mathrm{q}_{2}+\mathrm{q}_{4}+2 \mathrm{q}_{5}+\mathrm{q}_{6}=\mathrm{q}_{4}+\mathrm{q}_{6}+2 \mathrm{q}_{5}-\left(\mathrm{q}_{0}+\mathrm{q}_{2}\right)-2 \mathrm{q}_{1}
$$

The absolute value arithmetic is similar to the addition operations on the computer, so it is unified by the addition operations to calculate. The number of additions is $t_{1}=6$, the number of multiply is $t_{2}=2$ above formula. A pixel requires the calculation of four such templates, and coupled with the last difference calculation, therefore, a pixel needs the addition calculation of $6 \times 4+1=25$, the multiplication calculation of $2 \times 4=8$. It required a total of $25 \mathrm{~N}^{2}$ additions, $8 \mathrm{~N}^{2}$ multiplications, $155 \mathrm{~N}^{2}$ clock cycles for an $\mathrm{N} \times \mathrm{N}$ images.

\section{Conclusions}

In order to improve the deficiencies of traditional sobel operator, the paper proposed a method based on the absolute value of difference to calculate the gradient. The comparison of time-consuming for both algorithms is shown as Table 1 . It can be seen that the running time of improved sobel algorithm is approximately eight directions of sobel algorithm for $155 \div 280=0.5536$, saving of nearly half the time. Here only consider the complexity of template for edge detection, without considering the complexity brought about by other operations. The comparison of results for the edge detection is shown in Fig. 6, which is in the Matlab simulation environment for the same vehicle image. 
Table 1 Comparison of time-consuming for both algorithms

\begin{tabular}{|c|c|c|c|}
\hline $\begin{array}{c}\text { Edge } \\
\text { extraction method }\end{array}$ & $\begin{array}{c}\text { The number of } \\
\text { addition operations }\end{array}$ & $\begin{array}{c}\text { The number of } \\
\text { multiplications }\end{array}$ & $\begin{array}{c}\text { The number of clock } \\
\text { cycles required }\end{array}$ \\
\hline $\begin{array}{c}\text { Eight direction } \\
\text { sobel operator }\end{array}$ & $40 \mathrm{~N}^{2}$ & $16 \mathrm{~N}^{2}$ & $280 \mathrm{~N}^{2}$ \\
\hline $\begin{array}{c}\text { The improved } \\
\text { sobel operator }\end{array}$ & $25 \mathrm{~N}^{2}$ & $8 \mathrm{~N}^{2}$ & $155 \mathrm{~N}^{2}$ \\
\hline
\end{tabular}

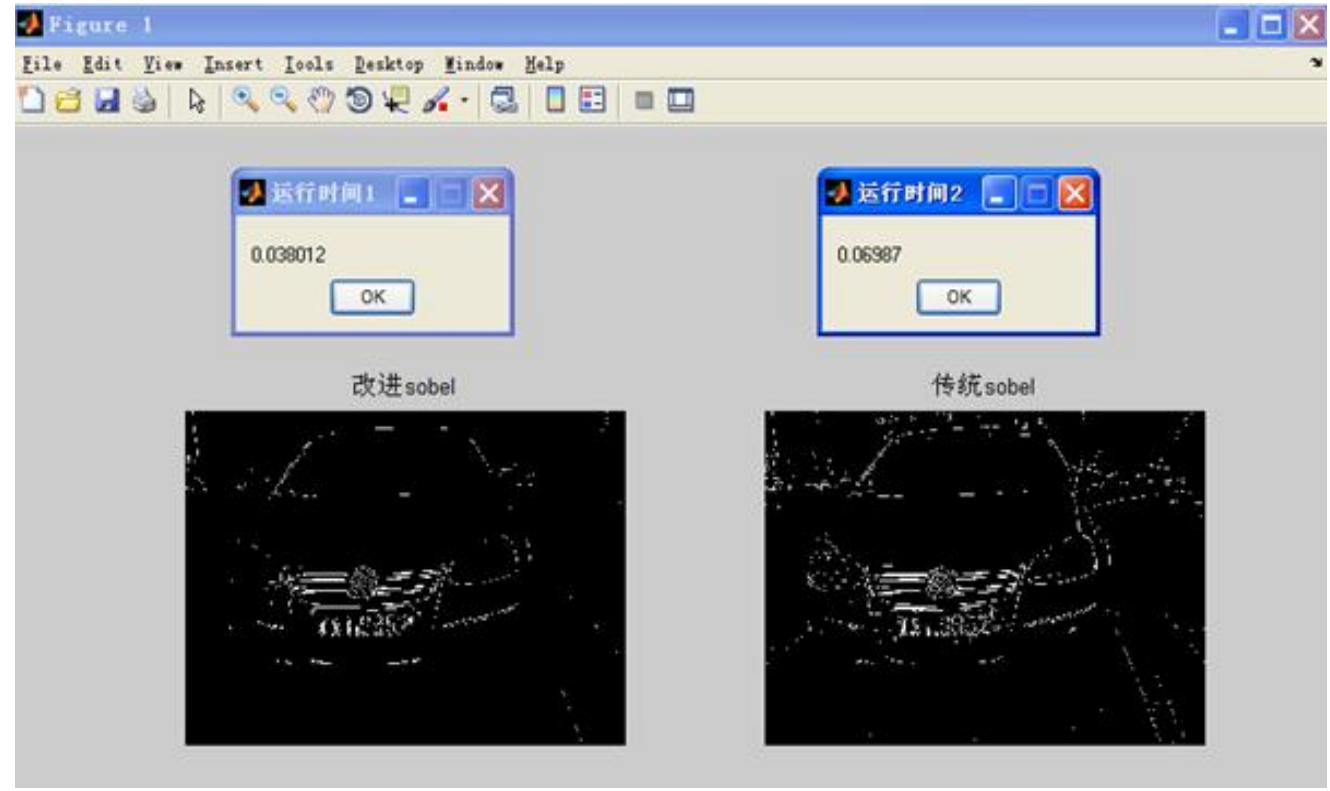

Figure 6. The comparison of results for the edge detection

It proved by the experiment that using the gradient algorithm to detect the edge in vehicle image can ensure the detection efficiency, reduce the computation, improve the processing speed, at the same time it has good noise immunity and lay the foundation for license plate location of follow-up.

\section{References}

[1] J.P.Deok: Pattern Recognition,Vol.28(2009) No.2,p.132..

[2] A.Alireza:Image and vision computing.,Vol. 27(2014) No.8,p.61.

[3] C.Liu, W.Sun: Journal of Guizhou University,Vol. 33(2013) No.5,p.33. (In Chinese)

[4] T.Wang and S.H.Quan: Computer Information,Vol. 24(2015) No.5, p.17. (In Chinese)

[5] Gangsaleisi: Digital Image Processing (MATLAB version) (Electronic Industry Press, Beijing 2008).p.10. (In Chinese).

[6] D.H.Shen,L.C.Zhang and E.Xu: Information Technology,Vol. 4(2015),P.43. (In Chinese)

[7] J.Sun:4th International Conference, AICI 2012(Chengdu, China, October 26-28, 2012). Vol. 7530,p.762-768.

[8] C.I.Gonzalez,P.Melin,J.R.Castro,O.Mendoza and O.Castillo:Soft Computing,Vol. 20(2016),No. 2,p.773-784.

[9] M.Y.Khomyakov:Pattern Recognition and Image Analysis,Vol. 22(2012) No.2,p.291-302.

[10]J.D.H.Pujar and D.S.Shambhavi:International Conference on Recent Trends in Business Administration and Information Processing,BAIP 2010(Trivandrum,Kerala,India,March 26-27,2010).Vol. 70,p. 91-95. 